\title{
EMOTIONAL LEARNING IN A SIMULATED MODEL OF THE MENTAL APPARATUS
}

\author{
Martin Fittner $^{1}$ and Christian Brandstätter ${ }^{2}$ \\ ${ }^{1}$ Institute of Computer Technology, Vienna University of Technology, Vienna, \\ Austria fittnerdict.tuwien.ac.at \\ ${ }^{2}$ Research Institute of Molecular Pathology (IMP) Vienna, Austria \\ brandstaetter@imp.ac.at
}

\begin{abstract}
How a human being learns is a wide field and not fully understood until now. This paper should give an alternative attempt to get closer to the answer how human beings learn something and what the relation to emotions is. Therefore, the cognitive architecture of the project "Simulation of Mental Apparatus and Applications (SiMA)" is used to fulfill two tasks. One is to give an answer to the question above and the other one is to enhance the functional model of the mental apparatus with learning. For that reason, the functions of the model are analyzed in detail for their ability to enhance them with a learning ability. The focus of the analysis lay on emotions and their impact on the ability to change memories in the model to determine a different behavior than without learning.
\end{abstract}

\section{KEYWORDS}

Learning, Emotion, Artificial General Intelligence, Simulation of Mental Apparatus and Applications (SiMA), Artificial Recognition System (ARS), Cognitive Architectures, Cognitive Automation, Psychoanalytically Inspired AI, Software Agents

\section{INTRODUCTION}

The target of the project Simulation of Mental Apparatus and Applications (SiMA) is the development of a holistic functional model of the human mind. It was founded by Dietmar Dietrich in 2000 [1] as a new attempt to overcome the lack of solutions for regulation of complex tasks in buildings that can't be solved with traditional algorithms. A bionic approach was used to develop a model of the human mind. As the best available holistic functional model [2] of the human mind the Metapsychology from Sigmund Freud [3] was taken as a framework for the model. This has been evaluated by [4] and [5] that stated that psychoanalysis fulfills the needed criteria of a unified holistic model of the mental apparatus. Like many other projects in cognitive science, the project moved to a multi-agent simulation to prove the functionality of the model. A methodology was developed [6] to transfer models from psychoanalysis and neurology into a holistic functional model. After 16 years, the model was enhanced and refined and remarkable results were reached [6]. But until now the functionality of learning was still missing. This paper should give the first outlook how learning can be integrated into the model with the focus on emotions.

David C. Wyld et al. (Eds) : CSITA, ISPR, ARIN, DMAP, CCSIT, AISC, SIPP, PDCTA, SOEN - 2017 pp. 11- 18, 2017. (C) CS \& IT-CSCP 2017

DOI : $10.5121 /$ csit.2017.70102 


\section{BACKGROUND AND RELATED WORKS}

To solve specific technical problems, a lot of learning algorithms have to be developed. They belong to the group of machine learning (reinforced learning, deep learning, neural networks, etc.) and showed remarkable results. But their strength is the solving of particular problems. The more they are used away from their application, the more they make failure in their calculations. For SiMA more general learning functions are needed to solve the problem of learning in a wider range. Therefore, machine learning can only be used in SiMA for particular data handling problems but not as a top-level learning model.

But there are more projects that deal with learning in the area of artificial general intelligence (AGI). These projects have the same target as SiMA to build a general-problem-solver (GPS). As a delegate of these projects, LIDA (Learning Intelligent Distribution Agent) should be discussed regarding their similarity to SiMA [7] and its functions for learning and emotions.

LIDA enhances its predecessor IDA (Intelligent Distribution Agent) by the ability to learn something. It combines several theories of the mind in a cognitive architecture [8]. In LIDA unconscious and conscious data processing is done due to the global workspace theory (GWT) that is used there. Learning is more conceptual but some parts are implemented like perceptual learning and episodic learning. In LIDA new information can only be stored (learned) when they first become conscious. Emotions and feelings are used as facilitators of learning [8] in the model.

\section{THE SIMA PROJECT}

The SiMA project uses the metapsychology as a framework for building a functional model of the human mind. This was done after an intensive search for a holistic unitary model of the human mental apparatus. To ensure a unified model different experts from other scientific disciplines are involved in the building of the model. Computer scientists are using such layer model now since the beginning of the first computers. The split of a system in its physical representation including dependencies and a pure data processing part without physics is a specialty of computer scientists. This was described together with the model in detail in [9]. So, with such a layer model, we can connect the data processing layers with the physical world. And as the physical world can be changed, as long as the interfaces to the new physics are adapted. So, it must be possible to run a human mental apparatus on a computer, as long as the hardware can provide enough power for the data processing.

The model is divided into 3 layers. Layer 1 (L1 in Figure 1) is the neural layer which handles signals from sensors and actors and represents the physical world of the model. The next layer is the neurosymbolic layer (L2 in Figure 1) where signals are transformed to neurosymbols as described by [10]. The neural and the neurosymbolic layer are not in the focus until now and realized as simple pass-thru functions. The third layer (L3 in Figure 1) was the starting point of SiMA. In this layer the human mental apparatus was located and divided into unconscious and conscious or preconscious data processing. The unconscious part was named as primary process and the conscious or preconscious part as secondary process. This separation has the advantage that a huge amount of data can be processed with fast automatic functions and only a small number of data is passed to the conscious processing part. This ensure that the system still can make decisions in nearly every situation. In Figure 1 the track view of SiMA is shown which is an abstract view of the model. 


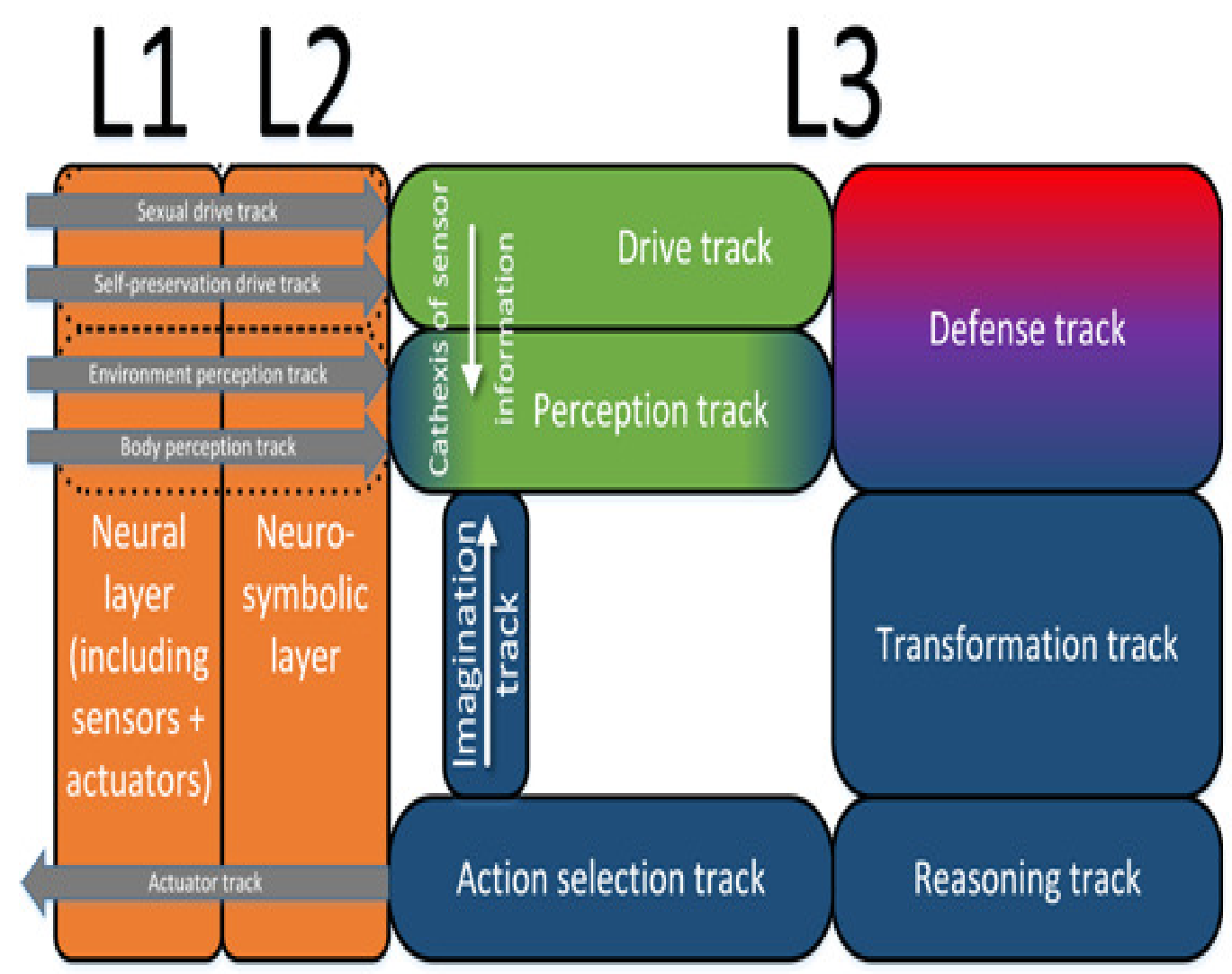

Figure 1. Functional model of SiMA in an abstract view [9]

\section{AN EMOTIONAL LEARNING MODEL}

To create a model for learning the following parts are needed:

- Memory

- Trigger for learning

- Change or creation of new data structure parts

How these parts are realized in SiMA and how they should be adapted to achieve a learning functionality are described in the following.

\subsection{The Memory Model}

The memory is located in the 1st layer of the SiMA model [9]. It corresponds to the multi storage model of Atkinson und Shiffrin [11]. A new interface is needed to access the memory from the functions. This can be seen as a separate data storage layer model to the data processing layer model of the functions as shown in Figure 2. 


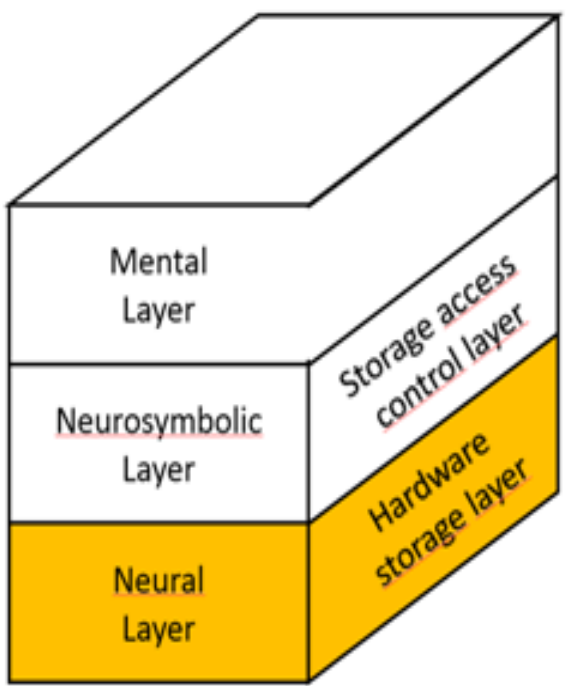

Figure 2. Memory model in SiMA

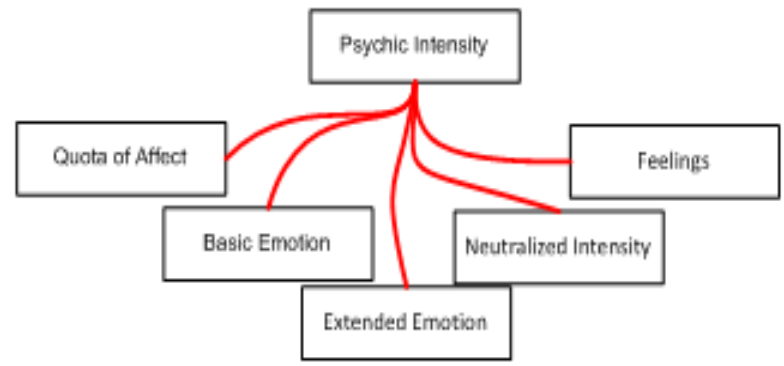

Figure 3. Psychic intensity and its components

The memory is further divided into short-term memory and long-term memory. An ultra-shortterm memory can't be used due to the missing implementation of the sensor functions, but in the model the values, that are handed from the simulator to the mind, are static for one processing cycle which goes a little bit in the direction of a sensory memory. For the hardware storage layer an ontology is used as a database and without production rules. A detailed description of the memory model can be found in [9].

\subsection{Emotions}

Emotions are one form of valuation in the SiMA model. All valuation terms are summarized as psychic intensity. As stated in [9] "In the SiMA project, psychic intensity is used as an umbrella term for all valuation quotas...Valuation ultimately serves to prioritize actions in order to mediate between the demands of the outside world (the own body and the environment) and the inner (psychic) needs (e.g. satisfying psychic and physiological needs within the environment, or adapting (psychic) wishes to the external circumstances)". In Figure 3 all valuations are pictured. Let us start with valuations of the secondary process. There we have feelings and neutralized intensity, where feelings are built up on emotions (basic and extended emotions) and neutralized intensity is created out of the quota of affect. So, the fundamentals of secondary process valuations reside in the primary process. There we have the quota of affect which is a valuation of the tension of sexual and self-preservation drives. The quota of affect was already equipped with a learning functionality. The outcome of the simulation shows that with the adaption of the quota of affect, that is connected with objects and actions, these objects or actions are more or less valuated and on the basis of these valuations the agents in the simulation decided how to interact with these objects. So, the same agent with the same personality in the same environmental setup chooses e. a. to eat an apple instead of a carrot just depending on the learned values of the quota of affect. As the valuation of the quota of affect is a part of the primary process it is unconscious. This matches perfectly with the human decision making when choosing something to eat. There is often no conscious reason why we sometimes choose this and sometimes that but there is an undeniable tendency that drags us to choose one of them. 


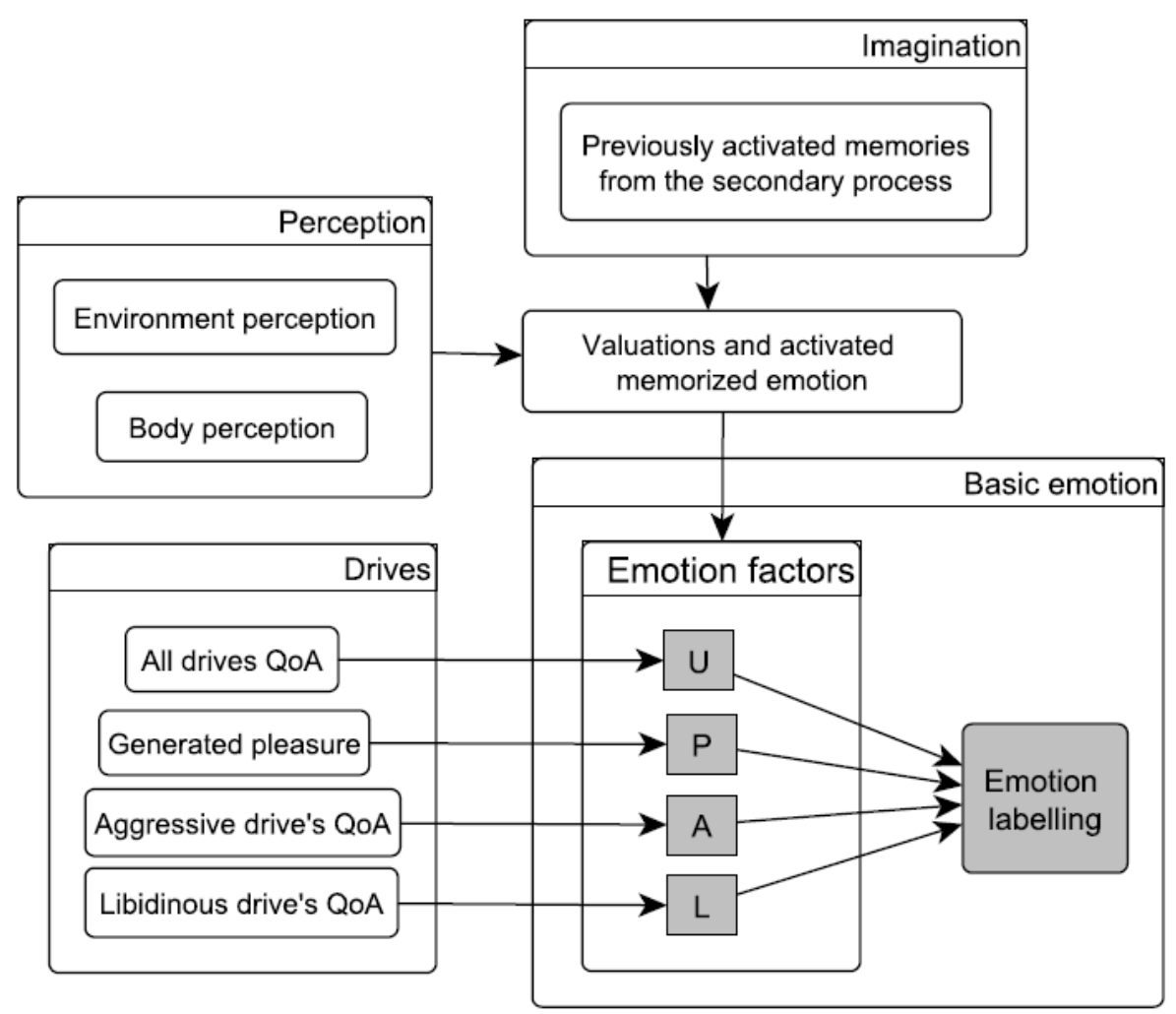

QoA ... Quota of Affect, U ... Unpleasure, P ... Pleasure, A ... Sum of aggressive drives' QoA , L ... Sum of libidinous drives' QDA

Figure 4. Creation of basic emotion in SiMA [6]

Emotions were separated into basic and extended emotions. Basic emotions are identical in every mammal and are formed out of four factors which can be seen in Figure 4. These factors evolve out of changes of the quota of affect of all drives. Basic emotions are further extended by conflicts of the defense mechanism. In this paper, basic emotions should be in the focus and therefore extended with a learning functionality to create new or change emotionally weighted memories of images. Images are snapshots of a current moment which combine all perceived information together.

\subsection{Exemplary Case}

The exemplary case [12] is used in the SiMA project to validate the correct function of new implementations. It also supports discussion of certain functionality of the model in an interdisciplinary collaboration. In this paper, the exemplary case should show how the change of experiences effects the agent's behavior.

The starting point of the exemplary case are two agents (Adam and Bodo) and two food sources (an apple and a carrot). At first, Adam perceived the situation and decided according to his hunger to go to the food source that attracts him more (the apple). His valuation of Bodo's bodily expressions doesn't lead to a change in his plan to eat the apple. As he reached the food source, he starts to eat. In the meantime, Bodo watched Adam and due to his predefined anger, he decided to beat Adam. When Bodo beats Adam, Adam perceives unpleasure and flees from Bodo. 


\section{SimUlation}

For the simulation, the above described exemplary case is used and simulated in a multi-agent environment based on MASON [13]. There are two simulation runs with different emotional valuations. The valuation is adapted manually until now but should be done by the agent in the near future. After the first run (Figure 5) the perceived unpleasure of Adam is captured with inspectors (numerical or graphical descriptions of certain internal states and decisions of the agent during the simulation) and used to adapt the emotional valuation of the bodily expressions of Bodo.

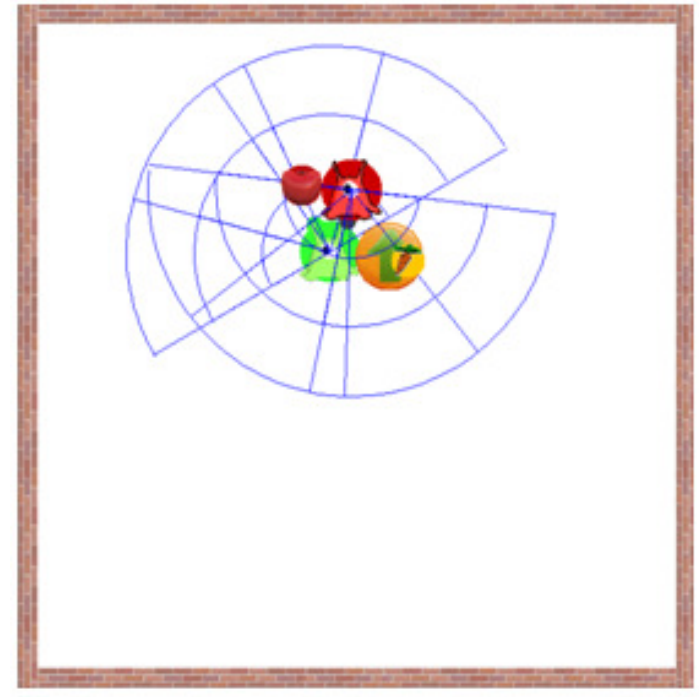

Figure 5. Learning phase

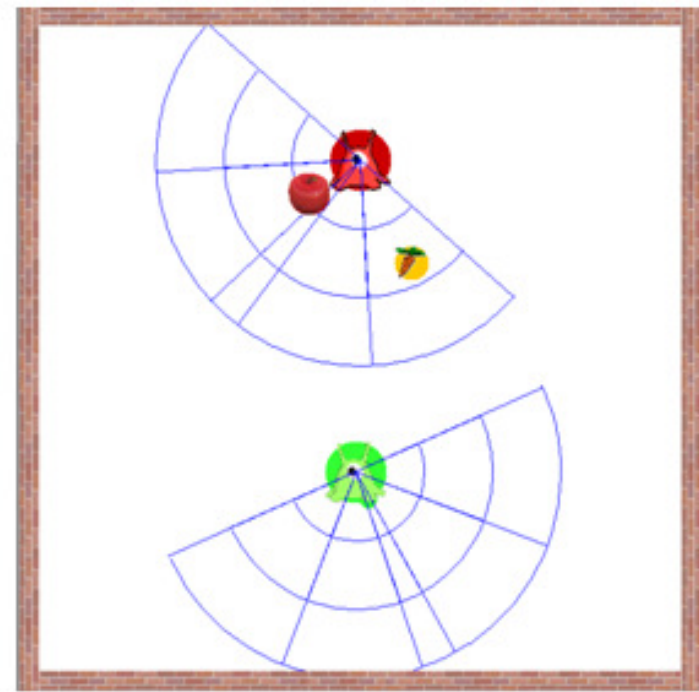

Figure 6. Behavior after Learning in 2nd run

After this adaption, the simulation is started again with the same parameters except the adapted emotional valuation. Now Adams emotions don't match with the plan to eat the apple and he decides to execute the second-best plan and flees (Figure 6).

\section{CONCLUSIONS AND OUTLOOK}

In contrast to the results of the learning with quota of affect valuations the valuations of emotions are unconscious, but with the transformation to feelings they become conscious. So the agent is also able to reason about the learned feelings that are a result of the changed emotions.

This paper shows a first attempt for the implementation of a learning functionality in regard to emotions. Now the change of emotional valuations must be implemented to produce the expected changes in memories. Then more complex exemplary cases can be created to show that the model is applicable for detecting dangerous situations. Therefore, the system could be used to valuate certain bodily expressions and actions of human beings. Of course, then we have to include a capable perception unit and the until now missing self-categorization.

\section{REFERENCES}

[1] Dietrich, Dietmar (2000) "Evolution potentials for Feldbus systems. In: Factory Communication Systems", Proceedings, 2000 IEEE International Workshop on Bd. 1, 2000, 145-146. - Invited Talk 
[2] Kandel, E. R. (1999) "Biology and the future of psychoanalysis: A new intellectual framework for psychiatry revisited" American Journal of Psychiatry, 505-524.

[3] Freud, S. (1915) "The Unconscious, volume XIV (1914-1916) of On the History of the PsychoAnalytic Movement, Papers on Metapsychology and Other Works. Vintag.

[4] Jakubec, M., Doblhammer, K., Fittner, M., Wendt, A.: Logical Thought Based on Word Presentations, in proceedings of EAPCogSci 2015, EuroAsianPacific Joint Conference on Cognitive Science, Torino, Italy, September 25-27, pp. 95-100, 2015.

[5] Dietmar Dietrich, D., Fodor, G., Zucker, G., Bruckner, D. (2009) "Simulating the Mind - A Technical Neuropsychoanalytical Approach” (2st ed.), Vienna: Springer.

[6] Schaat, S. (2016) "Simulation of Foundational Human Information-Processing in Social Context", Unpublished undergraduate dissertation, Vienna University of Technology.

[7] Wendt, A., Gelbard, F., Fittner, M., Schaat, S., Jakubec, M.: Decision-Making in the Cognitive Architecture SiMA. To be published at the 2015 Conference on Technologies and Applications of Artificial Intelligence (TAAI 2015), Taiwan, 2015.

[8] Franklin, S., Madl, T., D’Mello, S. Snaider, J., (2014) LIDA: A Systems-level Architecture for Cognition, Emotion, and Learning. IEEE Transactions on Autonomous Mental Development 6 (1), (pp. 19 - 41), IEEE.

[9] Dietrich, D., Brandstätter, C., Doblhammer, K., Fittner, M., Fodor, G, Gelbard, F., Huber, M., Jakubec, M., Kollmann, S., Kowarik, D., Schaat, S., Wendt, A., Widholm, R. (2015). Natural Scientific, Psychoanalytical Model of the Psyche for Simulation and Emulation; Scientific Report III, http://publik.tuwien.ac.at/files/PubDat_240983.pdf, Vienna University of Technology, Institute of Computer Technology.

[10] Velik, R. (2008). A Bionic Model for Human-like Machine Perception. Unpublished undergraduate dissertation, Vienna University of Technology, Institute of Computer Technology, Vienna.

[11] Atkinson, R. C., Shiffrin, R. M. (1968). Chapter: Human memory: A proposed system and its control processes. In Spence, K. W., \& Spence, J. T. The psychology of learning and motivation (Volume 2). New York: Academic Press. pp. 89-195.

[12] Schaat, S., Kollmann, S., Zhukova, O., Dietrich, D., Doblhammer, K. (2015) Examination of foundational AGI-agents in Artificial-Life simulations. Conference on Technologies and Applications of Artificial Intelligence (TAAI), Tainan, 2015, pp. 344-351.

[13] Luke, S., Cio-Revilla, C., Panait, L., Sullivan, K. (2004). Mason: A new multi-agent simulation toolkit. In Proceedings of the 2004 Swarmfest Workshop. Retrieved August 21, 2016, from http://cs.gmu.edu/ eclab/projects/mason/publications/SwarmFest04.pdf.

\section{AUTHORS}

Martin Fittner was born 1984 in Mistelbach (Austria), studied Embedded Systems at the University of Applied Science Technikum Wien in Vienna and finished with the degree Master of Science. He works as a senior developer for software and system design for automotive electronic control units at the Robert Bosch AG. His main topics are testing software for factory testing, customer bootloader programming and security protection of the system. He is a member of the SiMA (Simulation of the Mental Apparatus and Applications) team. Currently, he works on his dissertation at the Vienna University of Technology in the field of the SiMA project.

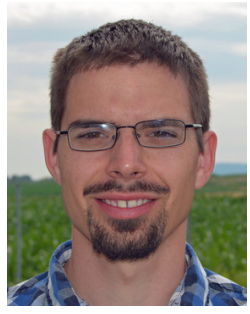


Christian Brandstätter was born 1979 in Tulln (Austria), studied Informatics and Informatics-Management at the Vienna University of Technology and finished both studies with the degree Master of Science. He works as System Administrator for general IT in a pharmaceutical research institute. His responsibility there is the IT infrastructure (network, storage, and security). Currently, he works on his dissertation and is a member of the Institute of Computer Technology at the Vienna University of Technology. Brandstätter, M.Sc. is a member of IEEE.

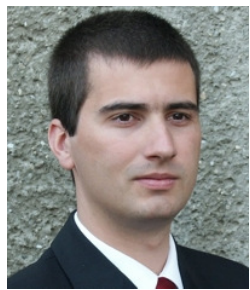

